Dtsch. Z. Philos., Berlin 40 (1992) 12, 1403-1417

\title{
Zur kritischen Theorie der Moral bei Adorno
}

\author{
Von GERHARD SCHWEPPENHÄUSER (Hannover)
}

Wer Äußerungen zum Thema Moralphilosophie aus dem Kreis der Begründer der Kritischen Theorie suchte, die geeignet erscheinen, über den systematischen Ort ethischer Reflexionen im Zusammenhang der Kritischen Theorie Aufschluß zu geben, sah sich bislang vor allem auf Gedanken von Horkheimer verwiesen. Dieser hatte im frühen Stadium der Entfaltung Kritischer Theorie das Konzept einer dialektischen Aufhebung derjenigen Intentionen, die in der idealistischen Moralphilosophie in falscher Gestalt aufbewahrt sind, durch befreiende gesellschaftliche Praxis vertreten. Die Idee der revolutionären Inauguration eines ebenso rationalen wie humanen gesellschaftlichen Bewegungsgesetzes schließt die materialistisch transformierte Vorstellung des Übergangs einer abstrakt-postulativen Moralität in konkrete Sittlichkeit ein. „Die Überwindung” der bürgerlich-idealistischen Moral, so Horkheimer 1936, „liegt nicht im Aufstellen einer besseren, sondern im Herbeiführen von Zuständen, unter denen ihr Daseinsgrund hinwegfällt. Die Verwirklichung der Sittlichkeit, eines menschenwürdigen Zustands, ist kein bloß seelisches, sondern ein geschichtliches Problem."1 Ohne von den frühen Intentionen grundsätzlich abzurücken, legte Horkheimer in späteren Jahren den Schwerpunkt darauf zu betonen, daß Kritische Theorie kein affirmatives Moralprinzip aufstellen könne. „Das jüdische Verbot, Gott darzustellen, und das Kantische, in intelligible Welten auszuschweifen, enthalten zugleich die Anerkennung dessen, eben des Absoluten, dessen Bestimmung unmöglich ist. Dasselbe gilt von der Kritischen Theorie, sofern sie erklärt, das Schlechte, zuvorderst in der Sphäre des Sozialen, dann aber auch in der des einzelnen Menschen, der des Moralischen, lasse sich bezeichnen, nicht jedoch das Gute. Der Begriff des Negativen, sei es das Relative oder das Böse, enthält in sich das Positive als seinen Gegensatz. Im Praktischen folgt aus der Denunziation einer Handlung als schlecht zumindest die Richtung der besseren Handlung. [...] Die kritische Analyse der Gesellschaft bezeichnet das herrschende Unrecht; der Versuch, es zu überwinden, hat wiederholt zu größerem Unrecht geführt. Einen Menschen zu Tode zu quälen ist Untat schlechthin, ihn, wenn möglich zu retten, menschliche Pflicht. Will man das Gute als den Versuch, das Schlechte abzuschaffen, definieren, so läßt es sich bestimmen. Eben dies ist die Lehre der Kritischen Theorie, jedoch das Gegenteil, nämlich das Schlechte durch das Gute zu definieren, wäre - selbst in der Moral - eine Unmöglichkeit."2

Um zu zeigen, daß es auch bei Adorno ein systematisch folgenreiches Konzept 
der kritischen Arbeit an moralphilosophischen Fragestellungen gibt, soll im folgenden ein bislang noch kaum erschlossener Aspekt seiner Philosophie vorgestellt werden. Es wird die These vertreten, daß bei Adorno Elemente moralphilosophischer Reflexion vorliegen, die zwar von ihm nicht zusammenhängend und vielfach nur indirekt entfaltet worden sind, sich aber zusammenführen lassen. ${ }^{3}$

Adornos untergründig präsente Moralphilosophie wird als „negative Moralphilosophie" bezeichnet. Das geschieht darum, weil Adornos "Reflexionen aus dem beschädigten Leben" - hier als übergreifende Charakteristik verstanden - sich kritisch-negierend $\mathrm{zu}$ diesem verhalten; weil Adorno sich weigert, affirmativ ein Moralprinzip aufzustellen, um seine Kritik zu fundieren; und schließlich darum, weil Adornos moralphilosophische Ausführungen über weite Strecken kritische Kommentare zur tradierten Moralphilosophie sind.

Die Moralphilosophie Adornos gilt es überhaupt erst zu konstruieren, weil es sie faktisch als solche nicht gibt. Das heißt, rekonstruiert wird ein nicht zur Ausführung gebrachter gedanklicher Zusammenhang als ganzer, so, wie er potentiell angelegt ist in Adornos Werk. Diese Rekonstruktion soll durch die Konstruktion ermöglicht werden.

Die Ausgangsfrage lautet, wie bereits angedeutet wurde, ob es einen moralphilosophischen Kern in Adornos Denken gibt, der sich im Zusammenhang herauspräparieren läßt. Auch wenn Kritische Theorie den esprit de systéme zu Recht unter Ideologieverdacht stellt, liegt ihr doch, das ist meine These, bei Adorno durchaus ein esprit systématique zugrunde, auch auf moralphilosophischem Gebiet.

Nun könnte man im gegenwärtigen philosophischen Kontext allerdings die Frage stellen, ob wir denn im aktuellen ethischen Diskurs überhaupt noch auf einen moralphilosophischen Ansatz angewiesen sind, der auf der Kritischen Theorie basiert, weil doch die Diskursethik in den letzten Jahren die Aufgabe übernommen habe, die von ihr beklagte vermeintliche Lücke eines normativen Fundaments der Kritischen Theorie zu schließen. Ich meine: Um zu einer kritischen Theorie der Moral zu gelangen, ist Adornos negative Moralphilosophie unerläßlich. Denn das, was die Diskursethik zu leisten imstande ist, ist nicht zu trennen von einer Reihe von Ausgrenzungen. Im folgenden werde ich einige Punkte nennen, an denen die Diskursethik immanent zu kritisieren wäre. ${ }^{4}$

Die Diskursethik beerbt den universalistischen Anspruch, den Kant der Moralphilosophie verliehen hat. Ebenso wie Kant tut sie das, indem sie die Form der Moralität einer Handlung und das moralisch Gesollte, das als rational begründbar definiert wird, ins Zentrum stellt. Um diese Momente stringent begründen zu können, rekurriert sie auf ein Moralprinzip, das allerdings, im Gegensatz zu Kant, intersubjektiv formuliert wird und am Maßstab konsensueller Wahrheitsfindung und Willensbildung orientiert ist. Der kategorische Imperativ wird depotenziert zur Verfahrensregel normenprüfender praktischer Diskurse; eine Regel, die entweder durch eine affirmative transzendentalpragmatische Letztbegründung (Apel) oder ex negativo durch den Aufweis ihrer praktischen Unhintergehbarkeit (Habermas) fundiert werden soll.

Um ihres formalen Prozeduralismus willen ist die Diskursethik zu Idealisierungen und Reduktionen genötigt: 1 . Sie hypostasiert die versachlichte Gestalt der 
Sprache, der sie die Idee kommunikativer Normativität entnimmt. 2. Das Moralprinzip wird nicht als philosophisch abgeleitetes aus einer Vielzahl bestimmter Negationen historischer Normen verstanden, sondern als transzendentale Gegebenheit, die in die intersubjektive Kommunikation "eingebaut" ist. 3. Es wird abgesehen sowohl vom Problem der theoretischen Unableitbarkeit der Idee der Moralität als auch von der Frage nach dem moralisch Guten selber und dem theoretisch nicht begründbaren praktischen Interesse der Menschen daran. 4. Unter Ausgrenzung der subjektiven, also vor allem motivationalen Aspekte des moralischen Gefühls wird in der Diskursethik der Schein eines möglichen Übergangs aus Rationalität in Moralität erzeugt. 5. Und schließlich werden in das Moralprinzip nicht die Bedingungen der Möglichkeit seiner Verwirklichung hineingenommen, weil die Diskursethik auf ein materiales Gerechtigkeitskonzept verzichten muß und damit auf die Kritik an einer antagonistischen Gesellschaft - eine Kritik, die selber eine moralische, normative Grundlage hat, weil sie Verhältnisse abgeschafft sehen will, in denen zentrale Postulate der Moralphilosophie, nämlich Freiheit und Selbstbestimmung der Individuen, nicht einzulösen sind. Die Diskursethik beharrt demgegenüber auf der Unparteilichkeit ihres "moral point of view".

Gleichwohl hält sie in der gegenwärtigen Debatte eine avancierte Position aufrecht, weil sie nicht bei einer privatistisch bleibenden Individualethik verharrt oder in die Aporien einer Güterethik gerät; und vor allem, weil sie starke Argumente gegen moralischen Relativismus und Skeptizismus ins Feld führen kann.

Sind die Reduktionen und der Formalismus der Tribut, den jede Moralphilosophie, die ein Universalisierungsprinzip aufstellt, für ihre Vernunftemphase zu entrichten hat? Oder ist eine moralphilosophische Position denkbar, die zwar das universalistisch-rationale Moment der Diskursethik teilt, sich aber nicht dem Zwang zu reduktionistischen Einschränkungen und zur Hypostasierung eines sprachlich-kommunikativen Moralprinzips unterwerfen muß? Meine These ist, daß Adornos Moralphilosophie diese Kriterien aufweist; unter anderem deswegen, weil sie auf ein affirmatives Prinzip verzichtet. Mit der Rekonstruktion von Adornos negativer Moralphilosophie liegt zwar noch keine "kritische Theorie der Moral" vor, aber immerhin ein weiterer Beitrag dazu.

Eine grundsätzliche Differenz zwischen Adorno und der Diskursethik sehe ich in folgendem Aspekt. Angeregt durch die empirischen Forschungen von Kohlberg nimmt Habermas an, daß auf dem Niveau postkonventioneller Moralität die höchsten erreichbaren individuellen und gesamtgesellschaftlichen Entwicklungsstufen der moralischen Urteilsbildung koinzidieren. Damit wird das Niveau bezeichnet, auf dem prinzipiengeleitete autonome Reflexion den "moralischen Gesichtspunkt" vertritt. Dieser ist gekennzeichnet 1. durch ein unparteiisches Interesse an Gerechtigkeit und Wohlergehen für alle handlungsfähigen Subjekte und 2. durch die Bereitschaft und Kompetenz zur intersubjektiven Konsensfindung über normative Streitfragen. Ontogenetisch bildet dieses Niveau den höchsten beobachtbaren Stand der moralischen Entwicklung; phylogenetisch wird sie gewissermaßen als regulative Idee diskutiert, als stets anzustrebende, aber vielleicht nie erreichbare Gestalt realisierter Rationalität. Die moralphilosophisch entscheidenden Annahmen sind also die Herausbildung universalistischer Moralprinzipien und die Überzeugung 
der Subjekte, daß über deren Geltungsanspruch nur im Diskurs entschieden werden könne.

Das „Projekt der Aufklärung“ in der Moderne besteht aus der Perspektive dieser Moralphilosophie darin, Moralprinzipien hieb- und stichfest abzusichern und darin, verbindliche Spielregeln des normativen Diskurses festzulegen. Die Frage ist, ob wir es uns leisten können, dabei weiterhin auf die dialektische Kritik der Prinzipien selber zu verzichten. Es ist eine Grundannahme der Kritischen Theorie, daß die universale Verwirklichung aufklärerischen Denkens dadurch hintertrieben wird, daß die Reflexion auf seine eigenen Schattenseiten unterbleibt. Wenn wir Begriffe wie Freiheit, Unparteilichkeit, Gerechtigkeit, Wohlwollen und Achtung unkritisiert übernehmen, dann übersehen wir etwas Entscheidendes: daß diese Begriffe einen historischen Index haben, der sie mit ihrer Negation verbindet, und zwar keineswegs nur äußerlich. Sie sind verbunden mit der Herrschaftsgeschichte der bürgerlichen Gesellschaft. Darum tragen sie stets auch das Gegenteil dessen in sich, wofür sie explizit einstehen. Freiheit ist bis heute insofern mit Unfreiheit verquickt, als sie für die Individuen substantiell wird als Freiheit, die eigene Arbeitskraft als Ware zu verkaufen. Unparteilichkeit kann in einer parteiisch verfaßten Gesellschaft Parteinahme bedeuten: nämlich für den Zustand, wie er nun einmal ist. Gerechtigkeit kann ,jedem das Seine“ bedeuten, und das heißt in den bestehenden gesellschaftlichen Verhältnissen leider immer auch: jedem nur das, was ihm nun einmal zusteht. Achtung vor dem anderen ist nie ganz zu trennen von der Leitlinie des Verhaltens, durch die wir sie erlernen: nämlich von Gehorsam.

Die Ambivalenz all dieser Vorstellungen muß eine kritische Theorie der Moral in ihre Reflexion mit hineinnehmen. Sie findet dafür ökonomisches, philosophisches und psychologisches Material bei Marx, Nietzsche und Freud, also, mit Alfred Schmidts Worten: bei den "großen Entzauberern der Moderne“5. Zusammen mit Kant sind das die wesentlichen Bezugspunkte für Adornos Ideologiekritik der Moralphilosophie. Was aber heißt hier Ideologiekritik? Es heißt nicht, daß die Moral mit dem Hinweis auf ihre repressiven Gestehungskosten auf den Müll geworfen würde. Es heißt auch nicht, daß Moralphilosophie per se für überflüssig oder schädlich erklärt würde. Den Beitrag einer philosophisch angeleiteten Ideologiekritik zur gegenwärtigen moralphilosophischen Diskussion möchte ich vielmehr so formulieren: Im historischen Interesse aller Menschen an der Verwirklichung einer rationalen und moralischen Regelung ihrer sozialen Interaktionen liegt es, daß die konstitutive Ambivalenz ethischer Intuitionen ins Bewußtsein tritt und auch dort bleibt. Denn nur was wir kennen, können wir verändern. In diesem Fall heißt das: Nur wenn wir stets um die Ambivalenz moralischer Kategorien wissen, können wir - vielleicht - verhindern, daß wir von ihren unterdrückenden Seiten beherrscht werden. Nur so können wir ihre befreienden Kräfte für die autonome Gestaltung unseres gemeinsamen Lebens auf diesem Planeten nutzen. Bei Adorno läßt sich viel darüber lernen, warum das emanzipatorische Potential prinzipiengeleiteter Normativität auch durch seine eigene innere Dialektik gefährdet wird. Nur wenn wir uns dieser Einsicht stellen, haben wir Chancen, dieses Potential auch aktualisieren zu können. Denn genau darum muß es uns gegenwärtig gehen; und ich meine, daß es auch Adorno vorrangig darum gegangen ist. 
Wenn man seine Ausführungen als Ideologiekritik der Moralphilosophie liest, macht man ihn keineswegs zu einem Vorgänger postmoderner Beliebigkeit auf ethischem Gebiet. Denn er hatte nicht die rationalitätsskeptische Denunziation bürgerlicher Moralphilosophie als pure Herrschaftsideologie im Sinn, wie wir sie heute zum Beispiel aus Gerd Kimmerles Attacken gegen Kant und die Diskursethik ${ }^{6}$ kennen. Adorno hat sich von Nietzsche, dem er so viel verdankt, an den Punkten abgegrenzt, an denen dieser die Moralität und ihre Kantische Reflexion abstrakt negativ behandelt und nicht den großen Fortschritt zur Humanität erkannt hat, den Adorno durch seine eigene kritische Lesart gerade erschließen wollte.

Adorno plante, ein moralphilosophisches Buch zu schreiben. Aber das wäre wohl kaum eine Moralphilosophie im traditionellen Sinn geworden. Traditionelle Moralphilosophie läßt sich (sowohl in der Antike als auch in der Neuzeit) als ein System kennzeichnen, das auf ersten, unhintergehbaren Annahmen, eben auf Prinzipien bzw. auf einem Prinzip aufgebaut ist. Hier setzt Adornos Kritik an. Man kann darin eine Entsprechung sehen zu seiner Kritik metaphysischer prima philosophia. Meine These lautet: Adornos Ausführungen zur Moralphilosophie können an einem theoretischen Leitfaden rekonstruiert werden, und dieser Leitfaden ist die Kritik einer praktischen Prinzipienphilosophie. Sie findet sich bei Adorno zwar nicht als theoretischer Ausgangspunkt expliziert. Aber sie bildet die unverzichtbare systematische Grundlage für die moralphilosophischen Implikationen seines veröffentlichten und unveröffentlichten Werks.

Traditionelle Moralphilosophie verhält sich keineswegs einfach affirmativ zur bestehenden Welt; im Gegenteil. Aber sie ist insofern affirmative Moralphilosophie, als sie Prinzipien aufstellt, die sie affirmiert. Adornos Ansatz besteht nun nicht in einer amoralistischen einfachen Negation dieser Systeme und ihrer Prinzipien. Es läßt sich zeigen, daß er sich vielmehr abarbeitet an den großen Entwürfen abendländischer Moralphilosophie, um ihr wesentliches Problem zu offenbaren. Diese Entwürfe stellen sich, so möchte ich zusammenfassen, kritisch zur bestehenden Welt, in der der einzelne Mensch leiden muß, aber sie reproduzieren in sich die antagonistischen Strukturen, die für das Leiden verantwortlich sind. Sie errichten eine hierarchische Ordnung von Prinzipien und daraus abgeleiteten Geboten, an deren Erfüllung der einzelne scheitern muß in einer Gesellschaft, die nicht vernünftig geordnet, sondern widersprüchlich ist. Die moralphilosophischen Systeme sind Ebenbilder der menschlichen Vernunft, doch die Wirklichkeit ist es nicht. Moralphilosophie eliminiert die Widersprüche aus ihrem System, aber nicht aus der Welt. Die Folgelasten dieses Konflikts hat der einzelne zu tragen, das Subjekt der Moralphilosophie. Das heißt, daß sich in der Moral ebenso wie in der Moralphilosophie Emanzipation und Repression untrennbar berühren. Unter diesem Aspekt steht Adornos Beschäftigung mit der Philosophie der Moral.

Im Begriff der Autonomie steckt bereits der Hinweis darauf, daß postkonventionelle, also vernunftgeleitete und selbstbestimmte Normativität der potentiell emanzipatorische Gehalt der Kantischen Moralphilosophie ist. Daran anknüpfend, vermittelt durch die von Marx entfaltete kritische Theorie des 19. Jahrhunderts, meldet die Kritische Theorie ihren normativen Anspruch an, den Horkheimer als das „Interesse an der Aufhebung des gesellschaftlichen Unrechts "7 bezeichnet. Adorno 
geht es nun darum, auf dem Gebiet der Moralphilosophie folgendes zu zeigen: Weder können stimmige, mit unbedingtem Geltungsanspruch verbundene Normen für das moralische Verhalten des Individuums unter den bestehenden gesellschaftlichen Bedingungen aufgestellt werden, noch ist es möglich, individuelle Verhaltensnormen unmittelbar daran auszurichten, was erst in einer "richtigen" gesellschaftlichen Totalität die Norm wäre. Führt der erste Weg zur Einebnung der kritischen Differenz zwischen dem, was ist, und dem, was sein soll, so endet der zweite in der Sackgasse ohnmächtigen Postulierens, das im Hegelschen Sinne abstrakt bleibt und, als Protest der Tugend gegen den Weltlauf, der Substantialität entraten muß. Hier stellt sich die Frage, wie Adorno sein uneingeschränktes Festhalten an der normativen Basis seiner Theorie - also das Interesse an der Abschaffung von Leiden und Unrecht, oder: an der Verwirklichung unverkürzter Humanität - mit der erklärten Unmöglichkeit vermittelt, normative Richtlinien des Handelns aufzustellen.

Der Versuch, diese Frage zu beantworten, muß Adornos Kritik moralischer Prinzipienphilosophie nachgehen. Sie richtet sich gegen den Anspruch, aus einem Moralprinzip das ihm entsprechende Handeln, ja einen ihm entsprechenden Gesamtzustand ableiten zu wollen. Insofern teilt Adorno Hegels Vorbehalt gegen „ein oberstes Moralprinzip [...], wie es jetzt genannt wird und woran man etwas Leeres hat, indem man alles zu haben glaubt".8 Aber er rekurriert gerade nicht wie dieser auf eine substantielle Sittlichkeit. Adornos Reflexionen gehen davon aus, daß die Suche nach einer "letztbegründeten“ Grundlage der Moral, also nach der Sphäre absoluter Ursprünge auf dem Gebiet der praktischen Philosophie, notwendig in Aporien führt. Der affirmative Nachweis eines Prinzips der Moral unterschlägt die innere Widersprüchlichkeit des Moralischen, die sich aus der Dialektik von allgemeinem und besonderem Interesse ergibt, welche die gesellschaftliche Basis von Moral ist. Diese Dialektik, die zuerst in der praktischen Philosophie des französischen Materialismus artikuliert wurde ${ }^{9}$, ist nach Adorno in die einzelnen moralischen Kategorien selber eingewandert. Darum ist es ihm auch nicht um einen soziologisierenden Reduktionismus zu tun. Er begreift moral-philosophische Reflexion als objektive geistige Bewegung, die dennoch von dem in ihr abgelagerten geschichtlich-gesellschaftlichen Gehalt nie abzutrennen ist. Ich vermute, daß sich der Rang moralphilosophischer Theoreme für Adorno daran bemessen haben dürfte, in welchem Maße sie durch ihre konsequente immanente Durchführung hindurch geselischaftlichen Gehalt transportieren.

Konkret heißt das, daß Adorno in moralischen und moralphilosophischen Systemen dem antagonistischen Verhältnis von Besonderem und Allgemeinem nachspürt. Er erkennt in den klassischen moralischen Geboten auf der einen Seite die Resultate der Anforderungen, die die gesellschaftliche Totalität stellt, etwa im Tötungsverbot; und auf der anderen Seite entschlüsselt er sie als verinnerlichte Resultate der Anforderungen des gesellschaftlich Partikularen, etwa in der protestantischen Arbeitsmoral und ihrer historischen Genese. Indem nun die Herrschaft partikularer Interessen sich historisch durch Kollektive hindurch manifestiert, werden besondere und allgemeine Ansprüche undurchschaubar vermengt. Dem Aufweis des Ineinanderspielens von apologetisch-ideologischen Inhalten und solchen, 
die von sich aus auf die Freisetzung individueller Autonomie in einem befriedeten Ganzen hinarbeiten, ist Adornos Auseinandersetzung mit der Moralphilosophie gewidmet. Seine eigene Position gewinnt Adorno, indem er diese kritisiert, nicht, indem er einen Gegenentwurf, ein eigenes ethisches System, aufstellt. Seine Kritik ist freilich nicht voraussetzungslos, sondern von dem normativen Interesse geleitet, von dem die Rede war.

Aus ihm ergibt sich Adornos doppelte Stellung zur Moral. Ihm geht es darum, das, was moralisch das Allgemeine ist, insoweit zu akzeptieren, wie es das Postulat individueller Freiheit und Autonomie zu formulieren erlaubt, und zugleich dasjenige an der moralischen Allgemeinheit zurückzuweisen, was - gewollt oder ungewollt - der Repression dient. Adorno hat hierbei keine säuberliche Trennung der emanzipatorischen Lehrstücke von den repressiven im Sinn. Die heuristische, analytisch-konstruktive Unterscheidung soll dem dialektischen Kern klassisch-moralphilosophischer Argumente gerecht werden. Diese dialektische Struktur ins Bewußtsein zu rufen heißt noch nicht, sie auflösen zu können. Aber nur das Bewußtsein, daß wir der moralischen Antinomie von Freiheit und Repression, von Autonomie und Heteronomie nicht entgehen können, kann nach Adorno ein erster Schritt sein, sich dem Wahrheitsgehalt dieser Autonomie zu stellen - ohne vor ihm ein für allemal zu kapitulieren. Mit Nietzsche versteht Adorno also „Moral [...] als Lehre von den Herrschafts-Verhältnissen [...], unter denen das Phänomen ,Leben' entsteht" ${ }^{10}$ Aber damit beschreibt Niezsche implizit, daß Moral und Herrschaft ineinander verschlungen sind, solange Vernunft nicht praktisch geworden und Autonomie zuinnerst mit ihrem Gegenteil verbunden ist.

Adorno mißt Moral und Moralphilosophie an ihrem eigenen Anspruch. Das ist der Maßstab seiner Kritik. Er stellt die Frage, inwieweit sie ihrem Begriff gerecht werden. Für die Antike bedeutet das, daß er der Problemstellung nachgeht, ob die theoretische Vermittlung der entstehenden Individualität mit der Totalität der Polis gelingt, bzw. warum sie nicht gelingen kann. ${ }^{11}$ Bei Kant wird zweierlei thematisiert: das Ineinander von Freiheit und Repression unter Bedingungen neuzeitlicher Verinnerlichung und die sozialphilosophische Perspektive universaler Autonomie, die in sich selber gebrochen ist. In der Kritik am abstrakt Postulativen einer hypostasierten Moralität folgt Adorno $\mathrm{Hegel}$ und hält diesem wiederum die Hypostasis einer nur vermeintlich substantiellen gesellschaftlichen Sittlichkeit vor. ${ }^{12}$ Und Nietzsches Kritik der idealistischen Abtrennung des Moralischen von seiner herrschaftsgeschichtlichen Abstammung geht, wie gesagt, zentral in Adornos moralphilosophische Reflexionen ein. Das hindert ihn jedoch nicht daran, den schwachen Punkt von Nietzsches Moralgenealogie bloßzulegen: die Verrechnung des normativen Geltungsanspruchs gegen die äußeren und inneren Gestehungskosten seiner Genesis, also, in Adornos Worten, die "abstrakte Negation der Moral ". ${ }^{13}$

Das Verhältnis der Kritischen Theorie Adornos zur Moralphilosophie läßt sich so charakterisieren: Moral ist in sich widersprüchlich, weil sie sowohl Freiheit als auch Unfreiheit in sich schließt. Die bestimmte Negation der Moral, um die es Adorno offensichtlich geht, soll nicht auf ihre Abschaffung hinauslaufen. Vielmehr wäre zu zeigen, wann und inwiefern Moral selber zwangsläufig unmoralisch wird. Was aber hat man sich unter dem von Adorno intendierten Verfahren der "bestimmten Negation" 
vorzustellen? Gemeint ist damit nicht die idealistische Stiftung neuer Positivität wie bei Hegel, sondern eine Negation der Negation im Marxschen Sinn. Die traditionelle Moralphilosophie in ihrer abstrakt-idealistischen Gestalt negiert die geschichtlichen und gesellschaftlichen Grundlagen, mit denen sie zusammenhängt. Diese Negation wird in Adornos Zugriff wiederum negiert, damit der produktive Gehalt der Moralphilosophie in einer kritischen Theorie der Moral aufgehoben werden kann.

Adornos moralphilosophisches Reflektieren verzichtet auf ein neues Moralprinzip. Es macht sich die humanitären, egalitären und universalistischen Intentionen der Typen von Moralphilosophie zu eigen, an denen es sich abarbeitet. Das geschieht nicht im Sinne einer bloßen Beschwörung alltagssprachlich formulierter humanitärer Ideale, sondern auf dem Wege einer spezifischen, dialektisch-philosophischen Aneignung. Dabei befindet sich Adorno sozusagen in einer doppelten Frontstellung. Er will einerseits jene Intentionen vor dem Vergessenwerden retten und sie gleichzeitig gegen ihre falsche Ausführung verteidigen. Andererseits soll gezeigt werden, daß in ihnen selber bereits, vermöge ihres unvermeidlich antagonistischen Charakters, der Grund dafür gelegt ist, daß sie sich gegen sich selber verkehren. Der kategorische Imperativ, das ist Adornos These, ist beides: radikal gewordene subjektiv-instrumentelle Rationalität und Vorgriff auf eine im objektiven Sinne vernünftig gewordene, befriedete Menschheit. Die von ihm vorgetragene Kritik am subjektiv-rationalen Charakter des Sittengesetzes zielt keineswegs auf dessen einfache Negation ab, sondern auf die bestimmte Negation des Aspekts falscher Einheit von kategorischem Imperativ und selbsterhaltender Vernunft. Mit dieser dialektischen Korrektur will Adorno den emanzipatorischen Gehalt der Kantischen Moralphilosophie zur Geltung bringen, die gerade im Aspekt der Differenz beider aufbewahrt ist. „Der Vorwurf, in der Objektivität des Sittengesetzes spreize einzig die subjektive Vernunft zum Absoluten sich auf, wäre subaltern. Kant spricht, fehlbar und entstellt, aus, was gesellschaftlich mit Grund $\mathrm{zu}$ fordern wäre. Solche Objektivität ist solange nicht in die subjektive Sphäre, nicht die der Psychologie und nicht die der Rationalität, zu übersetzen, sondern existiert zum Bösen und Guten getrennt von ihr fort, bis besonderes und allgemeines Interesse real zusammenstimmen. Das Gewissen ist das Schandmal der unfreien Gesellschaft."14

Adornos Konzept ist stimmig, weil er nicht von Moralphilosophie die Lösung realer Konflikte erhofft. Getreu den Prämissen der frühen Kritischen Theorie geht er vielmehr davon aus, daß nur durch gesellschaftliche Bewegung die ethischen Intuitionen in ihrer Ambivalenz einmal so zum Austrag gebracht werden könnten, daß sie nicht mehr bloß Reproduktion heteronomer Vergesellschaftungsverhältnisse wären, sondern Gegenstände autonomer - und zwar individueller und kollektiver Reflexion. Diese Perspektive erscheint Adorno zwar verstellt, aber er gibt sie gleichwohl nicht auf, und das ist für die Konstruktion seiner Moralkritik von wesentlicher Bedeutung.

Ein zentrales Motiv von Adornos negativer Moralphilosophie ist der "moralische Impuls". Die Wirklichkeit des Moralischen entzieht sich in Adornos Auffassung dem Ansinnen, sie hieb- und stichfest methodisch abzusichern. ${ }^{15} \mathrm{Mehr}$ als einen "Impuls" will Adorno nicht angeben, wenn nach der Instanz des "stellvertretenden" richtigen Handelns gefragt wird. 
In der Negativen Dialektik heißt es dazu: „Moralische Fragen stellen sich bündig [...] in Sätzen wie: Es soll nicht gefoltert werden; es sollen keine Konzentrationslager sein [...]. Bemächtigte aber ein Moralphilosoph sich jener Sätze und jubelte, nun hätte er die Kritiker der Moral erwischt: auch sie zitierten die von Moralphilosophen mit Behagen verkündeten Werte, so wäre der bündige Schluß falsch. Wahr sind die Sätze als Impuls, wenn gemeldet wird, irgendwo sei gefoltert worden. Sie dürfen sich nicht rationalisieren; als abstraktes Prinzip gerieten sie sogleich in die schlechte Unendlichkeit von Ableitung und Gültigkeit."16 Hier wird keinem ethischen Irrationalismus das Wort geredet. Es wird die Anstrengung unternommen, das im Begriff der Humanität gesetzte Fundament von Moralität normativ auszuweisen, ohne daß es als hypostasierter "Wert" verkündet würde; und ohne daß es an einem für Adorno fragwürdigen Ideal gemessen und um seine Gültigkeit gebracht würde: dem Ideal nämlich einer stringenten Deduzierbarkeit im Sinne instrumenteller Rationalität und einem damit verbundenen Anspruch auf unbedingte Gültigkeit, der gleichermaßen hybrid wie ohnmächtig ist. Der moralischnormative Impuls ist demzufolge schutzlos und nicht aus der Autorität einer verdinglichten Vorstellung von Rationalität allein herzuleiten. Er ist das prekäre Produkt des zivilisationsgeschichtlich errungenen Rationalitätsfortschritts, aber gebunden an die Sphäre dessen, was in Adornos materialistischer Philosophie als Bereich des Mimetischen und der auf leibhafter Erfahrung beruhenden humanen Solidarität zu bezeichnen wäre.

Im „moralischen Impuls", der sich angesichts des Grauens der Lager und der Folterer meldet, ist das Schopenhauersche Motiv des Mitleids wiederzuerkennen. ${ }^{7}$ Allerdings wird es bei Adorno nicht als fundamentale Triebfeder moralischen Handelns installiert, sondern aufgehoben im Zusammenspiel von humanem Impuls und kritischer Reflexion. Gleichwohl trägt Adornos Modell unverkennbar Züge des verborgenen Schopenhauerschen Materialismus ${ }^{18}$, der auf dem Gebiet der Ethik die lebendige Vorstellung fremden Leidens als Quelle uneigennütziger Handlungen erkennt und damit auf das mimetische Moment vorausdeutet, ohne das es nach Adorno keinen moralischen Impuls gäbe. Das Zerreißen des trügerischen, ideologischen Schleiers des Individuationsprinzips im identifikatorischen Mitleiden mit dem geschundenen Anderen, in dem wir uns selbst erkennen, ist bei Adorno in der mimetischen Basis der partikularen richtigen Handlung in der falschen Totalität aufbewahrt. Doch es ist nicht als neuplatonisch-mystische Erfahrung metaphysischer Wesenseinheit gedacht, sondern als eine Gestalt der negativen Erfahrung des gesellschaftlichen Unwesens, das alle Individuen unter seiner falschen Einheit zusammenzwingt.

Nach Adorno befinden sich moralischer Impuls und theoretisch-reflexives Begreifen antimoralischer Zustände insofern in einem antinomischen Verhältnis, als jede der beiden Reaktionsweisen für sich genommen ins Leere läuft. Erst im Zusammenwirken des auf Veränderung drängenden Impulses und der theoretischen Besinnung auf die Hindernisse, die seiner Umsetzung gebieterisch im Wege stehen und eine moralisch geleitete Praxis des isolierten Individuums blockieren, sieht Adorno die Chance von Moralität. Dieses Zusammenwirken beider Seiten der Antinomie kann für ihn freilich solange nur die Gestalt eines reflektierten Widerspruchs 
haben, wie in der gesellschaftlichen Wirklichkeit "das partikulare Interesse und die Menschheit auseinanderweisen".19 Das widersprüchliche Zusammenwirken von ,spontaner Regung' und ,theoretischem Bewußtsein' ist, so Adorno, „angesichts der realen Ohnmacht aller Einzelnen, der Schauplatz von Moral heute. “20

An dieser Stelle wird deutlich, daß sich Adornos moralphilosophisches Konzept nur explizieren läßt, wenn die systematische Verknüpfung mit seiner Ästhetik hergestellt wird. Der Rekurs auf Ästhetik im moralphilosophischen Zusammenhang hat also eine systematisch wichtige Funktion. Er kann zeigen, daß bei Adorno eine Zusammenführung ästhetischer und ethischer Erfahrungsgehalte stattfindet, die entfaltet werden muß, damit die Kategorie des moralischen Impulses erst ganz erhellt werden kann. Und umgekehrt ist, so lautet die These, im moralischen Impuls dessen ästhetische Dimension aufzudecken, damit das Verhältnis von Moralphilosophie und Ästhetik bei Adorno verständlich wird.

Im moralischen Impuls situiert Adorno das Ineinanderspielen und Zusammenwirken von leibhafter Erfahrung und Reflexion. Während die Sphäre der Reflexion hier in den Bereich der Moralphilosophie fällt, ist die Ästhetik für das Geschehen der leibhaften Erfahrung zuständig, weil sie es als einzige philosophische Disziplin explizieren kann. Ästhetik hat es mit der sinnlich vermittelten Erfahrung zu tun. In der Moderne ist sie befreit von ihrer klassisch-bürgerlichen Beschränkung auf die Rezeption des Schönen, vor allem des Kunstschönen, und nimmt damit zugleich Motive ihres disziplinären Ursprungs als Theorie reflektierter Sinnlichkeit wieder auf.

Die bürgerliche Ästhetik hatte die Vermittlung von Ethik und Ästhetik in der Erfahrung des Naturschönen aufgesucht. Nach Kant gibt es ein moralisches Interesse am Naturschönen. Die Verwandtschaft der ästhetischen und der intellektuellen Urteilskraft, die dem Geschmack und dem moralischen Gefühl zugeordnet werden, ist nach Kant konstitutiv für die Wahrnehmung des Naturschönen. ${ }^{21}$ Doch diese Vermittlung von Ethik und Ästhetik bleibt formal. Sie geht von einer Trennung der Zuständigkeitsbereiche im erkennenden und wahrnehmenden Subjekt aus. Wenn man ihre ästhetischen Implikationen transparent macht, zeigt Adornos Lehre vom moralischen Impuls dagegen, daß in der ethischen Erfahrung selber eine ästhetische Komponente stecken muß, damit sie sich überhaupt entfalten kann. Ästhetische Erfahrung, verstanden als "Einheit von Sinnlichkeit und Reflexion",22 ist also für moralische Erfahrung und ihre philosophische Theorie unabdingbar.

Im Gegensatz zur bürgerlichen Ästhetik also kann man mit Adorno moralphilosophisch relevante Einsichten sowohl in der Erfahrung des Naturschönen als auch in der des Kunstschönen gewinnen. In ästhetischer Erfahrung ist ihm zufolge der imaginative Vorgriff auf einen Zustand der Versöhnung von Mensch und Natur möglich, in dem der quasi-naturhafte Zwang instrumentalistischer Herrschaft gebrochen und Natur als humanisierte in zwanglose Kommunikation mit den Menschen als ihren Bestandteilen treten könnte. Im Begriff der Kulturlandschaft aktiviert Adorno ästhetische Naturwahrnehmung im Zeichen einer materialistischen Kritik am Naturbegriff bei Kant und Hegel.23 Die Utopie der Versöhnung wird freilich nicht affirmativ beschrieben, sondern steht, im Zeichen der Negativität, für die bestimmte Negation eines gewaltsamen Verhältnisses zur äußeren wie inneren 
Natur ein. Das Versprechen eines geglückten Lebens, das Adorno aus den Chiffren befriedeter Natur herausliest, ist, auch im Zuge der Diskussion über eine neue Naturästhetik und über die ethischen Verpflichtungen des Menschen der Natur gegenüber, erneut aktuell.

Ordnet man nun bei Adorno die Reflexion der Moralphilosophie zu und die leibhafte Erfahrung der Ästhetik, so zeigt sich, daß mit Hilfe der Einführung der Ästhetik die Reflexion im Ganzen des moralischen Impulses erst Konturen gewinnt. Dann läßt sich nämlich Adornos Theorie des moralischen Impulses so interpretieren: Nur wer ästhetische Erfahrungen (im weiteren Verständnis) machen kann, kann moralphilosophische Reflexion überhaupt fundieren. Somatisches Erleben und mimetische Erfahrung können somit als unverzichtbare Bestandteile des moralischen Impulses konzeptualisiert werden. Sie sind nicht kognitivistisch aufzulösen. Hier ergibt sich ein Berührungspunkt mit dem Begriff des moralischen Gefühls und der partiellen Rehabilitierung des Mitleidsmotivs in Horkheimers Überlegungen zu Materialismus und Moral.24 Vor allem aber ist vor diesem Hintergrund eine klare Abgrenzung möglich zwischen Adornos Überlegungen zum Verhältnis von ästhetisch-mimetischer und moralischer Sphäre und prämodern orientierten Versuchen, Ethik durch Ästhetik zu fundieren. 25

In der Erfahrung der Verstricktheit dessen, der die Vernichtungslager überlebt hat, kommen die beiden Grundthemen von Adornos Moralphilosophie zusammen: die Unmöglichkeit eines "richtigen Lebens" in einer falschen Totalität und der kategorische Imperativ, eine Wiederholung von Auschwitz zu verhindern.

Die negative Moralphilosophie unternimmt den Versuch einer Gratwanderung. Adorno will keine ,neue Moral‘ aufstellen, denn seine historisch ansetzende Ideologiekritik der Moral kommt zu dem Ergebnis: „Selbst die Normen, welche die Einrichtung der Welt verdammen, verdanken sich deren eigenem Unwesen. Alle Moral hat sich am Modell der Unmoral gebildet und bis heute auf jeder Stufe wiederhergestellt." 26 Aber er weiß, daß Kritische Theorie der Normativität bedarf und insofern das Erbe der Moral anzutreten hat. Und das ist nach Adorno auch möglich, denn Moral hat keineswegs nur ideologischen, herrschaftsstabilisierenden Charakter, sondern kann "den Gesellschaftszustand selbst angreifen". ${ }^{27}$ In Ethik steckt "kraft der Antithese des Seinsollenden zum Seienden"28 utopisches Potential. Adorno will es aktualisieren und für die Kritische Theorie aneignen, indem er die Hypostasierung des moralischen Impulses in affirmativen ethischen Systemen kritisiert.

Systemkritik ist aber, wie eingangs bereits gesagt wurde, nicht gleichbedeutend mit unsystematischem Denken. In dem für die Konstruktion seiner Philosophie als Ganzer bedeutsamen Text Der Essay als Form ${ }^{29}$ wird das Programm eines theoretischen Verfahrens formuliert, das sich nicht an einem fixierten System orientiert, dessen Begriffe deduktiv oder induktiv auf das Material anzuwenden sind, das es zu erschließen gilt, sondern das als Verfahren seine immanente Logizität entwickelt, indem es sich den Objekten seiner theoretischen Arbeit anmißt. Insofern ist das Verfahren nicht unsystematisch, sondern anhand von Kriterien organisiert, die sich angeben lassen, aber nur, indem sie in der Auseinandersetzung mit dem untersuchten Gegenstand selbst gewonnen werden, und nicht indem sie als vorher schon 
fertige Kategorien verstanden werden, zu deren Überprüfung der Gegenstand herangezogen wird.

Im Hinblick auf die Thematik meiner Untersuchung möchte ich die These formulieren, daß es einen gemeinsamen systematischen Fluchtpunkt gibt, auf den sich Adornos moralphilosophische Reflexionen beziehen lassen. Es ist der Versuch der Artikulation moralphilosophischer Erfahrung. Im Essay soll die Artikulation von Erfahrung ihre erkenntnisstiftende Form, die sie für Adorno einem methodischformalen, wissenschaftlichen Erkenntnisideal überlegen macht, darum gewinnen können, weil der Essay imstande ist, durch das substantielle Eindringen in geistige Gebilde eine Konkretion von Erfahrung zu ermöglichen. Entsprechend können wir in den Elementen von Adornos negativer Moralphilosophie, die kein Prinzip aufstellen wollen, aber gleichwohl prinzipiell ansetzen, die Arbeit an der Artikulation der moralischen Erfahrung erkennen, die für Adorno offenbar den Vorrang gehabt hat vor einem auf Geltungsfragen bezogenen ethischen Begründungsverfahren.

Eine weitere systematisch zentrale moralphilosophische Kategorie neben dem "moralischen Impuls" ist der ,neue kategorische Imperativ', der den Menschen befehle, „ihr Denken und Handeln so einzurichten, daß Auschwitz nicht sich wiederhole, nichts Ähnliches geschehe."30 Schaut man sich dieses normative Fundament von Adornos Moralphilosophie genauer an, das er aber nicht als Moralprinzip verstanden wissen will, dann zeigt sich: Adorno grenzt die Frage nach der diskursiven Begründbarkeit aus seiner ethischen Reflexion aus. Er tut dies, um die Evidenz des humanen moralischen Impulses nicht zu relativieren.

Das Fehlen einer Theorie der Begründung und der Geltung moralischer Sätze oder Normen ist, aus der Perspektive einer Theorie der Artikulation moralischer Erfahrung betrachtet, nicht unbedingt als ein Defizit zu bewerten. Es ist - paradox formuliert - implizit sehr wohl begründbar: nicht eine unbeabsichtigte Leerstelle, sondern Ergebnis einer theoretischen Entscheidung, die ihrerseits begründungsfähig ist. Darin ließe sich ein Schritt des Übergangs vom Schulbegriff der Philosophie zu ihrem Weltbegriff im Sinne Adornos erkennen.

Der Hintergrund dafür ist, daß Erfahrung für Adorno Erfahrung von Auschwitz bedeutet. „Erfahrung soll nicht heißen, persönliche Erfahrung des Augenzeugen, sondern Erfahrung des Bewußtseins", schreibt Claussen. "Diese Erfahrung des Bewußtseins hat Adorno zum Ausgangspunkt gesellschaftskritischer Wahrnehmung gemacht." 31 Ist nach Auschwitz noch eine "politische Moral“ möglich? Das fragte Söllner kürzlich in Abwandlung des berühmten, oft mißverstandenen Satzes von Adorno. ${ }^{32}$ Diese Fragestellung korrespondiert mit Adornos moralphilosophischen Intentionen. „Nur in der Reflexion auf Auschwitz könnte der Bann gebrochen werden, den eine entfesselte instrumentelle Vernunft über die Kultur der Moderne verhängt hat" 33 - so kennzeichnet Dubiel die Aufgabenstellung Kritischer Theorie. Für Adorno wäre die Fixiertheit auf formale Begründungsverfahren demgegenüber sicherlich eine Fortsetzung des instrumentellen Bannes gewesen. Denn, wie wiederum Claussen formuliert: "Auschwitz erzwingt ein Erinnern ohne Begründung, eine andere, neue Form des Nachdenkens über gesellschaftliche Beziehungen." 34

Die philosophische Intention einer Artikulation moralisch relevanter Erfahrung 
kann als das Unabgegoltene bezeichnet werden, das den Gehalt von Adornos Moralphilosophie ausmacht. Diese Intention hat sich in seiner eingreifenden, auf aktuelle Probleme der Gegenwart gerichteten Essayistik manifestiert. Dem geistigen Kampf gegen gesellschaftliche Heteronomie, gegen Tabus und Borniertheiten, gegen die Wiederkehr des verdrängten faschistoiden Potentials und gegen die Unterhöhlung politischer Demokratisierung durch ökonomische und sozialpsychologische Faktoren liegt bei Adorno das Interesse an Autonomie und Freiheit zugrunde.

Das verweist auf die Sphäre der Konkretion und Artikulation der moralischen Erfahrung, der Form, die sie in seinen Essays gewinnt. Eine erneute Lektüre der Essays von Adorno könnte, dieser Grundlagen eingedenk, Adorno als moralischen Essayisten oder als essayistischen Moralisten entdecken, dessen moralphilosophische Argumentationsbasis nicht intuitiv oder evokativ bleibt, sondern argumentative Stringenz aufweist, die nachprüfbar ist.

Auf Adornos Beitrag zur moralphilosophischen Diskussion sollten wir daher nicht verzichten. Das gilt besonders in einer Gegenwart, in der sich die Barbarei in unserem Land erneut zu formieren beginnt. Gegen Rassismus und Pogrome können wir mit Moralphilosophie zwar nichts ausrichten, aber diese Phänomene sollten unser Bewußtsein dafür schärfen, daß die moralische Regression mit dem gesellschaftlichen Bewegungsgesetz zusammenhängt, das wir nach wie vor selber produzieren, ohne es rational zu beherrschen.

Dr. phil. Gerhard Schweppenhäuser, Universität Hannover, Philosophisches Seminar, Welfengarten 1, W- 3000 Hannover 1

\section{Anmerkungen}

1 Max Horkheimer: Egoismus und Freiheitsbewegung, in: ders.: Gesammelte Schriften, Bd. 4, hrsg. v. A. Schmidt, Frankfurt/M. 1988, S. 83.

2 Ders.: Notizen 1950 bis 1969, in: ders.: Notizen 1950 bis 1969 und Dämmerung. Notizen in Deutschland, hrsg. v. W. Brede, Frankfurt/M. 1974, S. 215.

3 Dieser Aufsatz ist eine Zusammenfassung einiger Hauptgedanken meiner Dissertation: Elemente einer negativen Moralphilosophie bei Theodor W. Adorno, Hamburg 1992. Daher werden Resultate vorgestellt, aber nicht hergeleitet. Die hier fehlende argumentative Entfaltung enthält die genannte Arbeit. Ansätze zur Behandlung des Themas finden sich bei Robert Schurz: Ethik nach Adorno, Basel/Frankfurt/M. 1985. Diese bislang einzige Monographie zum Thema ist insgesamt jedoch unsystematisch strukturiert, was zur Folge hat, daß sich die Studie bisweilen in der Fülle ihres Materials verliert, das anscheinend kaum konstruiert und mitunter nur assoziativ verarbeitet ist. Daher trägt sie leider wenig bei zur Klärung der hier untersuchten Fragen. Produktive Anregungen zur Auseinandersetzung mit der Frage der moralischen Normativität bei Adorno enthält der Aufsatz von Klaus Günther: Dialektik der Aufklärung in der Idee der Freiheit. Zur Kritik des Freiheitsbegriffs bei Adorno, in: Zeitschrift für philosophische Forschung, Bd. 39 (1985), Heft 2, S. $229 \mathrm{ff}$. Neben diesem Aufsatz sind folgende Arbeiten zu nennen: Anke Thyen: Negative Dialektik und Erfahrung. Zur Rationalität des Nichtidentischen bei Adorno. Frankf./M. 1989, und Hauke Brunkhorst: Theodor W. Adorno. Dialektik der Moderne, München 1990. Sie behan- 
deln das Thema Moralphilosophie zwar nur am Rande, enthalten aber einige wertvolle Anregungen. Inzwischen, nach Abschluß meiner Studie, hat Mirko Wischke zwei einschlägige Arbeiten vorgelegt: Betroffenheit und Versöhnung. Die Grundmotive der Moralphilosophie von Theodor W. Adorno, in: Deutsche Zeitschrift für Philosophie, 40. Jg., Heft 8/1992, S. 900 ff. und: Kritik der Ethik des Gehorsams. Adorno und Nietzsche - Zur postmodernen Ästhetisierung der Ethik, in: Ästhetik und Kommunikation, Heft 79 (21. Jg.), Oktober 1992, S. 111 ff. Sie enthalten eine Interpretation von Adornos Moralphilosophie, die von der hier vertretenen erheblich differiert. Es steht also eine Diskussion über Adornos Moralphilosophie an, die in diesem Aufsatz noch nicht geführt werden kann; er ist auch als ein Anstoß zu dieser Diskussion zu verstehen.

4 Dabei stütze ich mich auf folgende Arbeiten: Albrecht Wellmer: Ethik und Dialog, Frankfurt/M. 1986; Herbert Schnädelbach: Max Horkheimer und die Moralphilosophie des deutschen Idealismus, in: ders., Vernunft und Geschichte, Frankfurt/M. 1987, S. 207ff. und Axel Honneth: Diskursethik und implizites Gerechtigkeitskonzept, in: Moralität und Sittlichkeit, hrsg. v. Wolfgang Kuhlmann, Frankfurt/M. 1986, S. 183ff.

$5 \mathrm{Vgl}$. Alfred Schmidt: Die in Naturgeschichte verstrickte Menschheit, in: Krise und Kritik, hrsg. v. G. Schweppenhäuser u. a., Lüneburg 1987, S. 10.

6 Vgl. Gerd Kimmerle: Vom kategorischen Imperativ des gebrochenen Begehrens, in: Vorschrift und Autonomie, hrsg. v. G. Gamm u. G. Kimmerle, Tübingen 1989, S. $103 \mathrm{ff}$.

7 Max Horkheimer: Traditionelle und Kritische Theorie, in: ders.: Kritische Theorie, hrsg. v. A. Schmidt, Bd. II, Frankf./M. 1968, S. 190.

8 Georg Wilhelm Friedrich Hegel: Vorlesungen über die Geschichte der Philosophie II, in: ders.: Werke, Bd. 19, Frankfurt/M. 1971, S. 106.

9 Vgl. dazu Günther Mensching: Totalität und Autonomie, Frankf./M. 1971, 6. Kapitel, S. $178 \mathrm{ff}$.

10 Friedrich Nietzsche: Jenseits von Gut und Böse, Aph. 19 (in: ders.: Werke in drei Bänden, hrsg. v. K. Schlechta, Bd. II, München 1977, S. 583).

11 Vgl. Theodor W. Adorno: Gesammelte Schriften, hrsg. v. R. Tiedemann (im folgenden: GS), Frankfurt/M. 1970-1986, Bd. 10, S. 769.

12 Vgl. Theodor W. Adorno: GS, Bd. 6, S. 211 ff. und ders.: GS, Bd. 10, S. 764f. sowie GS, Bd. 6, S. $295 \mathrm{ff}$.

13 Nach Adorno ist es das Problem auch der Moralkritik Nietzsches, „daß es ihm am Begriff der bestimmten Negation gefehlt hat [...]. Bei Nietzsche [...] handelt es sich um den Versuch, aus Verzweiflung über das einmal als schlecht Erkannte eine neue Ordnung, neue Werte [...] aus dem Nichts gleichsam zu beschwören und entgegenzuhalten." (Theodor W. Adorno, Max Horkheimer, Hans-Georg Gadamer: Über Nietzsche und uns, in: Horkheimer: Gesammelte Schriften, Bd. 13, hrsg. v. G. Schmid Noerr, Frankfurt/M. 1989, S. 116.

14 Theodor W. Adorno: GS, Bd. 6, S. 272.

15 Das wird denn auch von einigen Interpreten der Negativen Dialektik beklagt; vgl. Michael Theunissen: Negativität bei Adorno, in: Adorno-Konferenz 1983, hrsg. v. L. v. Friedeburg u. J. Habermas, Frankf./M. 1983, S. 50ff.

16 Theodor W. Adorno: GS, Bd. 6, S. 281.

17 Vgl. Arthur Schopenhauer: Preisschrift über die Grundlage der Moral, Teil III, in: ders.: Zürcher Ausgabe, Bd. 6, Zürich 1977, S. 143ff.

18 Vgl. dazu: Alfred Schmidt: Schopenhauer und der Materialismus, in: ders.: Drei Studien über Materialismus, München/Wien 1977.

19 Theodor W. Adorno: GS, Bd. 16, S. 19.

20 Ders.: GS, Bd. 6, S. 282.

21 Vgl. Immanuel Kant: Kritik der Urteilskraft, $\S 42$. 
22 Vgl. Heinz Paetzold: Ästhetik der neueren Moderne, Stuttgart 1990.

23 Vgl. Theodor W. Adorno: GS, Bd. 7, S.101ff.; vgl. dazu: Paetzold: Neomarxistische Ästhetik, Bd. II, Düsseldorf 1974, S. $26 \mathrm{ff}$.

24 Vgl. Max Horkheimer: Materialismus und Moral, in: Zeitschrift für Sozialforschung, Bd. 2, S. $182 \mathrm{ff}$.

$25 \mathrm{Vgl}$. zum letztgenannten Aspekt Klaus Günther: Das gute und das schöne Leben, in: G. Kimmerle u. G. Gamm (Hrsg.): Ethik und Ästhetik, Tübingen 1990, S. $11 \mathrm{ff}$.

26 Theodor W. Adorno: GS, Bd. 4, S. 210.

27 Ders.: GS, Bd. 8, S. 263.

28 Ders.: GS, Bd. 8, S. 272.

29 Vgl. ders.: GS, Bd. 11, S. 9 ff.

30 Ders.: GS, Bd. 6, S. 358.

31 Detlev Claussen: Grenzen der Aufklärung, Frankf./M. 1987, S. $10 f$.

32 Alfons Söllner: Vortrag, gehalten in Lüneburg am 8. 7. 91.

33 Helmut Dubiel: Kommentar zu Leo Löwenthals "Individuum und Terror", in: Zivilisationsbruch, hrsg. v. D. Diner, Frankfurt/M. 1988, S. 29.

34 Claussen, a. a. O., S. 24. 


\section{Ethik der Gabe}

\section{Denken nach Jacques Derrida}

Herausgegeben von Michael Wetzel und Jean-Michel Rabate

Acta humaniora - Schriften zur Kunstwissenschaft und Philosophie

1992. Ca. 330 Seiten $-170 \mathrm{~mm} \times 240 \mathrm{~mm}$

Hardcover ca. 48,- DM

ISBN 3-05-002370-8

Dieser Sammelband vereint einige Vorträge, die auf dem Kolloquium aus Anlaß des 60. Geburtstages von Jacques Derrida im Dezember 1990 in Royaumont gehalten worden sind. Sie wenden sich vor allem der ethischen Dimension im Werk des Philosophen zu.

\section{Aus dem Inhalt:}

Giorgio Agamben: Pardes. Die Schrift der Potenz - Michel Lisse: „Zu lesen geben“ - Elisabeth Weber: Schwarze Tränen, Tintenspur - Hent de Vries: Das Schibboleth der Ethik. Derrida und Celan - Jean-Michel Rabaté: Von der Gabe des Gedichts zur Vergebung für den Dichter? Der Sagetrieb Ezra Pounds - Jacques Derrida: Wenn es Gabe gibt oder „Das falsche Geldstück“-Rene Major: Die Wahrung der Gabe - Ulla Haselstein: Die Gabe der Wilden. Mary Rowlandsons captivity narrative - Jochen Hörisch: Dekonstruktion des Geldes - Catherine Malabou: Wozu das Leben sparen wollen, wo nichts mehr ist? - Bernard Stiegler: Verkehrte Aufzeichnungen und photographische Wiedergabe - Mireille CalleGruber: Die Gabe des Sehens - Michael Wetzel: Liebesgaben. Streifzüge des literarischen Eros-Manfred Schneider: Das Geschenk der Lebensgeschichte: die Norm-Geoffrey Bennington: Mosaique. Politik und Grenzen der Dekonstruktion - David Wills: Dem Buchstaben nach geben-Dieter Lesage: Eine Erschütterung des Eigennamens - Ulrike Dünkelsbühler: Kein Recht Auf-Gabe. Der KörperEffekt des Polyneikes - Paola Marrati: Der Traum und die Gefahr. Wo sich die sexuelle Differenz verliert

Verzeichnis der Schriften Derridas

Bestellungen richten Sie bitte an Ihre Buchhandlung oder an den

\section{Akademie Verlag}

Ein Untemehmen der VCH-Veriagsgruppe

Leipziger StraBe 3-4 - Postfach 1233 - D-1086 Berlin 